\title{
Research on evaluation of service quality of third-party E-commerce platform
}

\author{
Wang Xinping ${ }^{\mathrm{a}}$, Ding manli $\mathrm{b}^{*}$ \\ School of management \\ Xi'an University of Science and Technology, \\ Shaanxi Silk Road Quality Research Institute \\ Xi'an, 710054, China \\ a34443679@qq.com \\ bjas_dingmanli@163.com \\ Ding man li*
}

Keywords: E-commerce; Service quality; Evaluation

\begin{abstract}
Regards a large number of market share of third-party e-commerce platform, this paper combined with the classic research results of service quality evaluation, and analyzed the service quality elements of the third-party e-commerce platform. Besides, this paper constructed the service quality evaluation system, and based on fuzzy comprehensive evaluation method, the third-party e-commerce platform service quality evaluation model was established.
\end{abstract}

\section{Introduction}

With the rapid development of e-commerce in China and the relative backwardness of related service quality theories, this paper bases on the environment of e-commerce, selects e-commerce platform as the research background of the industry, and puts forward the research questions of this article - evaluation of the service of e-commerce platform Quality ${ }^{[1]}$.

\subsection{Research methods}

Based on the current situation of e-commerce platform service in our country and domestic and international theoretical circles' research on the quality of e-service, this article mainly establishes the scope of the research object based on the combination of theoretical analysis and empirical analysis, qualitative analysis and quantitative analysis, as well as characteristics of e-commerce platform based on the transaction process to establish a service quality evaluation model ${ }^{[1]}$.

\subsection{The main point of view and innovation}

This paper argues that good quality of service has become a major strategy for e-commerce enterprises to be competitive. The innovation of this article is to combine the traditional classic SERVQUAL service theory with fuzzy comprehensive evaluation in system engineering to evaluate the service quality of e-commerce platform ${ }^{[4]}$.

\section{Service Evaluation Model}

\subsection{SERVQUAL}

Palau Surriman, Zeit Hamel and Berry developed SERVQUAL as a tool for assessing service quality based on five dimensions of service quality. It is an effective tool for gap analysis. Mainly from five dimensions of service quality analysis: tangible, reliability, responsiveness, assurance, empathy ${ }^{[2]}$.

\subsection{Rating system}

25 specific survey items are designed according to SERVQUAL five dimensions. 
Table1 Service quality question form of third-party e-commerce platform

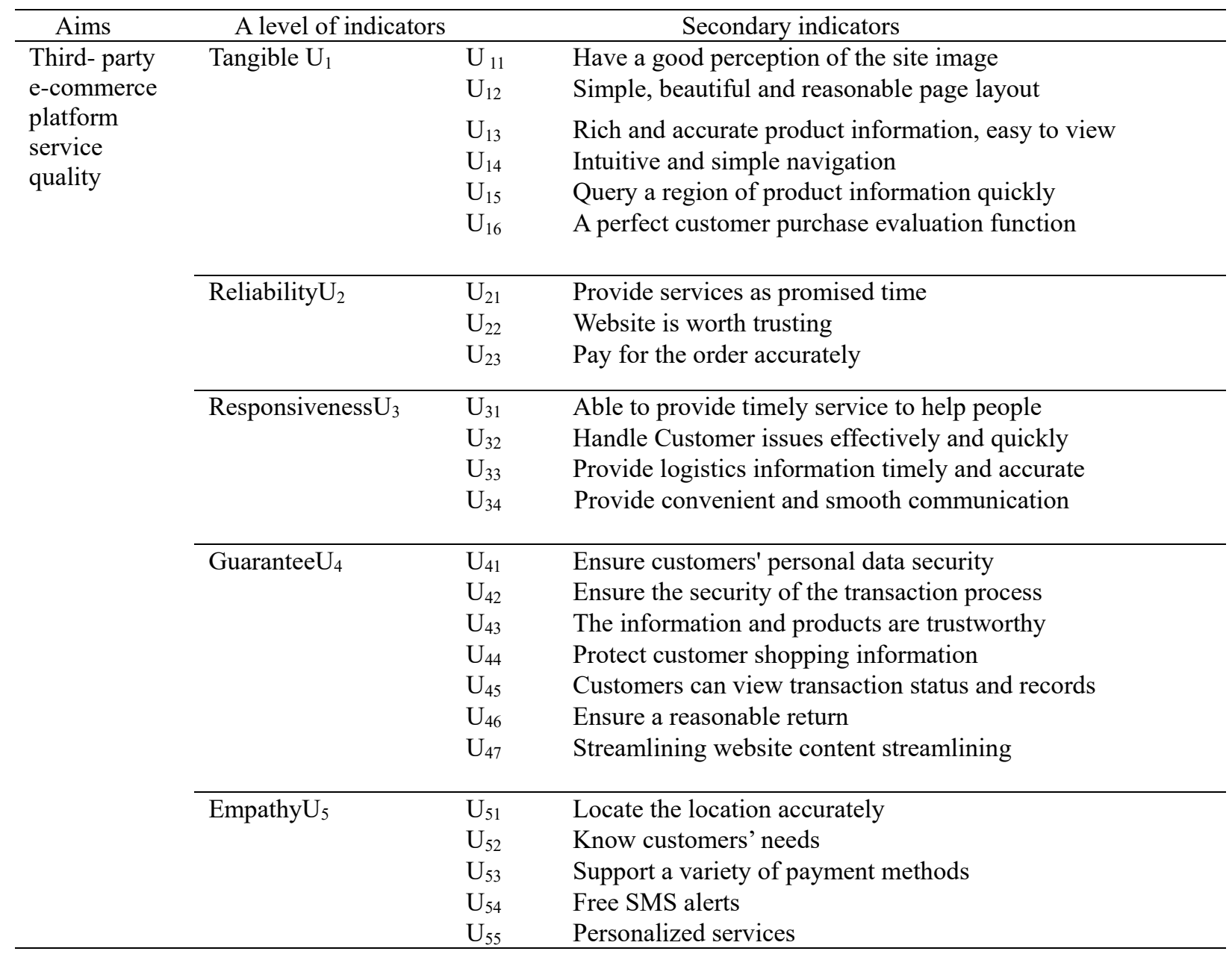

\subsection{Evaluation methods and procedures}

In the evaluation of these issues, it is difficult to use an accurate figure to express their feelings, the evaluation results can only be a vague concept, the description of the uncertainty is ambiguity. In the third-party e-commerce service quality platform for evaluation, the fuzzy comprehensive evaluation model steps are as follows ${ }^{[4,5,6]}$ :

\subsubsection{Set of factors}

The evaluation indexes of service quality of the third-party e-commerce platform are divided as shown in the above table. The total quality of service goal $U$ is divided into five first-level indicators Ui, and each first-level indicator is respectively divided into corresponding second-level indicators Uij. Specifically:

$$
\mathrm{U}=\left(\mathrm{U}_{1}, \mathrm{U}_{2}, \ldots, \quad \mathrm{U}_{\mathrm{n}}\right)
$$

" $n$ " represents the number of first-level indicators, $n=5$ in this study, and $m$ represent the number of second-level indicators of $\mathrm{U}_{\mathrm{i}}$.

\subsubsection{Set up a comment set}

A comment set is a set of various general evaluation results that the evaluator may make for the object of evaluation. Generally, $\mathrm{V}=\left\{\mathrm{v}_{1}, \mathrm{v} 2, \ldots \mathrm{vn}\right\}=(5,4,3,2,1)=$ Very satisfied, more satisfied, general, not satisfied, very dissatisfied), the vi represents a variety of elements of the overall evaluation results.

2.3.3 Statistics, determine the single factor evaluation membership vector, forming membership matrix

The degree of membership refers to the possibility that multiple evaluation bodies make a $\mathrm{v}_{\mathrm{j}}$ rating on a certain evaluation object in terms of $U_{i}$. Usually, the proportion of the number of people who 
evaluate $U_{i j}$ as a certain grade $V_{j}$ to the number of evaluation persons is the corresponding membership degree $\mathrm{r}_{\mathrm{ijk}}$. Medium $\mathrm{k}=5$ Evaluation factor i membership matrix vector.

$$
\begin{array}{r}
\mathrm{R}_{\mathrm{i}}=\left(\mathrm{r}_{\mathrm{i} 1}, \mathrm{r}_{\mathrm{i} 2}, \ldots, \quad \mathrm{r}_{i m}\right), \quad \mathrm{i}=1,2,3, \ldots \mathrm{n}, \sum_{j=1}^{m} r_{i j k}=1 \\
\text { Membership matrix } \mathrm{R}=\left(\mathrm{R}_{1}, \mathrm{R}_{2}, \ldots, \mathrm{R}_{\mathrm{n}}\right)^{\mathrm{T}} \\
\text { Can be expressed as: }\left[\begin{array}{cccc}
r_{i 11} & r_{i 12} & \cdots & r_{i 1 k} \\
r_{i 21} & r_{i 22} & \cdots & r_{i 2 k} \\
\cdots & \cdots & \cdots & \cdots \\
r_{i m 1} & r_{i m 2} & \cdots & r_{i m k}
\end{array}\right],
\end{array}
$$

\subsubsection{Set up a factor weight set}

Among the factors, the degree of importance of each factor is different. In order to reflect the degree of importance of each factor, a certain weight should be given to each factor. The set of weights of each factor is called the factor weight set.

$$
\mathrm{W}=\left\{\mathrm{w}_{1}, \mathrm{w}_{2}, \ldots \mathrm{w}_{\mathrm{n}}\right\}, \sum_{i=1}^{n} W_{i}=1
$$

\subsubsection{Determine the fuzzy comprehensive evaluation matrix $B$}

Comprehensive evaluation of $\mathrm{U}_{\mathrm{i}}$, you can get:

$$
\begin{aligned}
B_{i} & =W_{i} \bullet R_{i} \\
& =\left(W_{i 1}, W_{i 2}, \cdots, W_{i m}\right)\left[\begin{array}{cccc}
r_{i 11} & r_{i 12} & \cdots & r_{i 1 k} \\
r_{i 21} & r_{i 22} & \cdots & r_{i 2 k} \\
\cdots & \cdots & \cdots & \cdots \\
r_{i m 1} & r_{i m 2} & \cdots & r_{i m k}
\end{array}\right] \\
& =\left(b_{i 1}, b_{i 2}, \cdots, b_{i k}\right)
\end{aligned}
$$

After the comprehensive evaluation of each quality factor $U_{i}$ contained in $U$, we get $B_{i}, i=1,2, \ldots$ n. Respectively, for the normalization of $\mathrm{B}_{\mathrm{i}} \mathrm{U}$ to obtain a comprehensive evaluation matrix $\mathrm{B}$ :

$$
\mathrm{B}=\left(\mathrm{B}_{1}^{\prime}{ }_{1}, \mathrm{~B}_{2}^{\prime}, \ldots, \mathrm{B}_{\mathrm{n}}^{\prime}\right)^{\mathrm{T}}
$$

\subsubsection{U fuzzy comprehensive evaluation}

It is known that the comprehensive evaluation matrix of $U$ is $B$ and the weight vector is $W$, so the comprehensive fuzzy evaluation result of $U$ is:

$$
\mathrm{Z}=\mathrm{W} \cdot \mathrm{B}=\left(\mathrm{W}_{1}, \mathrm{~W}_{2}, \ldots, \mathrm{W}_{\mathrm{n}}\right) \cdot\left(\mathrm{B}^{\prime}{ }_{1}, \mathrm{~B}_{2}^{\prime}, \ldots, \mathrm{B}_{\mathrm{n}}^{\prime}\right)^{\mathrm{T}}=\left(\mathrm{Z}_{1}, \mathrm{Z}_{2} \ldots \mathrm{Z}_{\mathrm{k}}\right)
$$

Last third party e-commerce platform fuzzy comprehensive evaluation of service quality score $\mathrm{P}$ :

$$
\mathrm{P}=\mathrm{ZV}^{\mathrm{T}}
$$

For the evaluation platform, the value of $\mathrm{P}$ with the $\mathrm{V}$-set rating of the comment set can be compared to obtain the client's satisfaction with the platform; for evaluation proponents, the value of P from big to small can be arranged in order. It can be learned that the highest score of platform service is optimal.

\section{Conclusions}

In this paper, the method of combining theoretical analysis with model analysis is used systematically and normatively. First, with the theoretical analysis, in the context of the development of the Internet, emphasizes the importance of the quality of service of e-commerce platform. Secondly, this paper uses the traditional classic SERVQUAL service evaluation methods, designed 25 different issues according to their own characteristics of e-commerce platform. Then uses the fuzzy comprehensive evaluation method in system engineering to evaluate and calculate the service quality of different e-commerce platforms. By using this research method, we can avoid 
the objective reality that e-commerce platform service cannot be accurately evaluated, and draw the difference of service quality more accurately, so as to provide guidance to the e-commerce platform to improve service quality.

\section{Acknowledgement}

*supported by the national natural science foundation project (71403204) and the key research base planning project (14JZ027) of the philosophy and social sciences of universities in Shaanxi province.

\section{References}

[1] Chen Jing hua. C2C e-commerce platform for service quality and customer loyalty [D]. Xiamen University, 2009.

[2] Thomas Forster. Quality Management: The Integrated Approach [M]. Beijing, 2005: 242-253.

[3] Wang Mingming, Zhao Guowei. B2C mobile e-commerce service quality evaluation system [J]. Technology Management Research, 2015,35 (03): 142-145.

[4] Cao Erli. Service quality analysis of third-party B2B e-commerce platform [J] .Business Research, 2010 (06): 213-216.

[5] Wang xinping. Management System Engineering.[M].Beijing: Machinery Industry Press,2011:159-163.

[6] Wu qizong. System Engineering.[M].Beijing: Beijing Institute of Technology Press, 2006:226-231 